\title{
$\begin{array}{ll}\text { Research Square } & \begin{array}{l}\text { Preprints are preliminary reports that have not undergone peer review. } \\ \text { They should not be considered conclusive, used to inform clinical practice, } \\ \text { or referenced by the media as validated information. }\end{array}\end{array}$
}

\section{Upregulation of B3GNT3 Correlates With Poor Prognosis and Decreased Immune Cell Infiltration In Pancreatic Adenocarcinoma}

\author{
Long-Jiang Chen \\ Renmin Hospital of Wuhan University \\ Lu-Lu Zhai \\ Renmin Hospital of Wuhan University: Wuhan University Renmin Hospital \\ Wei Wang \\ Renmin Hospital of Wuhan University: Wuhan University Renmin Hospital \\ Lun Wu \\ Renmin Hospital of Wuhan University: Wuhan University Renmin Hospital \\ Li-Chao Yao \\ Renmin Hospital of Wuhan University: Wuhan University Renmin Hospital \\ Feng Xiang \\ Renmin Hospital of Wuhan University: Wuhan University Renmin Hospital \\ Wei-Bo Li \\ Renmin Hospital of Wuhan University: Wuhan University Renmin Hospital \\ Zhi-Gang Tang ( $\nabla$ tzgwhu@163.com ) \\ Renmin Hospital of Wuhan University: Wuhan University Renmin Hospital
}

\section{Research Article}

Keywords: B3GNT3, Pancreatic adenocarcinoma, prognosis, tumor-infiltrating immune cells, TCGA database

DOI: https://doi.org/10.21203/rs.3.rs-218028/v1

License: (9) This work is licensed under a Creative Commons Attribution 4.0 International License. Read Full License 


\begin{abstract}
While previous studies have suggested that B3GNT3 is associated with tumorigenesis and progression of several tumors, its expression level and clinical significance in pancreatic adenocarcinoma (PAAD) remains unclear. Our study aimed to investigate the role of B3GNT3 in PAAD. B3GNT3 RNA sequencing and clinicopathological data were collected from the TCGA, GEO and GTEx databases. We assessed the expression and prognostic value of B3GNT3 in PAAD using R program and attached packages. Additionally, we investigate the correlation between B3GNT3 expression and tumor-infiltrating immune cells using CIBERSORT and the "correlation" module of GEPIA. Finally, gene set enrichment analysis (GSEA) was used to elucidate B3GNT3 related signaling pathways in PAAD. Results showed that B3GNT3 expression was significantly higher in tumor tissues compared to normal tissues ( $P$ $<0.05)$. Increased B3GNT3 expression was correlated with advanced histologic grade and stage ( $\square$ Vs $\square)$. Patients with high B3GNT3 expression had a worse OS ( $\mathrm{HR}=1.713, \mathrm{P}=0.0005)$. Moreover, a negative correlation between increased B3GNT3 expression and immune infiltrating level of naive B cells, CD8 T cells, and CD 4 memory activated T cells was revealed by CIBERSORT analysis. Then, further analysis verified the correlation using the "correlation" module of GEPIA. Finally, GSEA suggested that functional enrichment of B3GNT3 was mainly involved in pathways in cancer, p53 signaling pathway, TGF beta signaling pathway, catabolic and transport processes of proteins, etc. Collectively, these results suggested that overexpression of B3GNT3 might affect the infiltration of immune cells and could act as a potential prognostic biomarker of PAAD.
\end{abstract}

\title{
Introduction
}

Pancreatic adenocarcinoma (PAAD) is one of the most lethal solid malignancies and the fourth leading cause of cancer-related deaths, accounting for an estimated 57,600 new cases and 47,050 deaths yearly in the United States. Seriously, PAAD is increasing in both incidence and mortality and tends to become the second leading cause of cancer death in the USA in the next twenty to thirty years $[1,2]$. Due to the absence of specific symptoms and its aggressive nature, majority of patients diagnosed with pancreatic adenocarcinoma present with either unresectable or metastatic disease[3]. Despite advances in diagnostic measures, perioperative management, surgical techniques and systemic therapies for advanced disease, most patients newly diagnosed with PAAD will die within 6 months and the 5-year survival rate is less than 10\%. Moreover, the pathogenesis of PAAD is still inadequately understood now. Risk factors previously well illustrated to be associated with development of PAAD included inherited predisposition to pancreatic cancer, obesity, new-onset diabetes, tobacco use, and chronic pancreatitis[4-8]. All the factors may arouse abnormal alteration of gene expression and culminate in development of overt pancreatic adenocarcinoma. With the advance of transcriptomesequencing and functional genomics analysis, we availably assessed alterations of gene expression and underlying roles in carcinogenesis and therapeutics of pancreatic adenocarcinoma.

B3GNT3 (Beta-1,3-N-Acetylglucosaminyltransferase 3) is a protein coding gene and belongs to the $\beta 3$ GlcNAcT family. It is located on chromosome 19q13.1 and comprises of three exons. Commonly, B3GNT3 is predominantly expressed in colon, stomach, gall bladder, duodenum and small intestine, and lowly expressed in other tissues including pancreas, liver, lung and brain. In recent years, increasing evidences implicated that the $\beta 3$ GIcNAcT family appeared to be involved in tumorigenesis and progression of several tumors. A molecular analysis of 120 resected lung cancer confirmed that B3GNT3 overexpression was correlated with poor prognosis of lung cancer patient and could be negatively regulated by miR-149-5p[9]. Similarly, significant upregulation of B3GNT3 was also observed in early-stage cervical cancer. In the 196 cases of cervical cancer patients, higher expression of B3GNT3 indicated more lymph node metastasis and poorer prognosis[10]. In addition, for patients with neuroblastoma, increased B3GNT3 expression could suppress malignant cell migration and invasion by modulating mucin-type 0-glycosylation and signaling in neuroblastoma cells[11]. However, the relationship between B3GNT3 and PAAD has not been well elucidated.

In the current study, we performed bioinformatics analysis to investigate the expression of B3GNT3 in PAAD using data from the public domain. We analyzed the relationship between B3GNT3 expression and clinical parameters of PAAD and correlated them with patients' overall survival. Then, we also assessed relationship of B3GNT3 with tumor-infiltrating immune cells in tumor tissues and B3GNT3-related biological pathways involved in PAAD. Taken together, our findings provide new insights for a potential correlation as well as a possible mechanism of B3GNT3 in PAAD.

\section{Materials And Methods}

\section{Data collection from public databases}


Publicly available datasets of PAAD patients, with gene expression RNAseq profiles and corresponding clinical information, were downloaded from the Cancer Genome Atlas (TCGA) and Genotype-Tissue Expression project (GTEx) using the UCSC Xena browser ( https://xenabrowser.net/)[12]. The clinical information included age, gender, histologic grade, pathologic stage, tumor location, radical resection, and survival time. Expression profiles from the GSE62452 dataset were obtained from the GEO database (http://www.ncbi.nlm.nih.gov/geo/). Duplicates and datasets without normal adjacent tissues or with small sample sizes in GEO database were excluded. Then, these raw data were furtherly transformed and merged for analysis using Perl and $\mathrm{R}$ program. Due to the public availability of data on above datasets, additional restriction or limitation was not required for this study.

\section{Expression and survival analysis of B3GNT3}

R limma package was performed to investigate the Gene expression of B3GNT3 in different datasets[13]. Firstly, the B3GNT3 expression values of 167 normal pancreas tissues from GTEx, 4 tumor-adjacent normal tissues and 176 PAAD tissues from TCGA, and 61 tumor-adjacent normal tissues and 69 PAAD tissues from GSE62452 were calculated and presented. Secondly, the associations between expression of B3GNT3 and clinicopathologic parameters including Age, Gender, Race, Family history of cancer, History of chronic pancreatitis, History of diabetes, Alcohol consumption, Tumor location, Maximum tumor dimension, Histologic grade, T status, N status, M status and stage were analyzed using TCGA data for the available clinical information. Finally, the Kaplan-Meier method was employed for the analysis of overall survival based on B3GNT3 expression level. In addition, B3GNT3 expression and other clinicopathological factors were subjected to Cox proportional hazards regression analysis to evaluate the prognostic value. All the analyses were proceeded and plotted in R packages and the Log-Rank was utilized to test the significance among different survival curves.

\section{Enumeration of tumor infiltrating immune cells}

CIBERSORT is a robust method for characterizing cell composition of complex tissues from their gene expression profiles [14]. Tumor infiltrating immune cells (TIIC) abundance can therefore be precisely estimated via this way. The concentration abundance profile of 22 leukocyte subtypes in PAAD tissues of TCGA was obtained using the 'CIBERSORT' R package, with a cut-off P-value $<0.05$. According to median value of the B3GNT3 expression level, all samples were divided into high-expression and low expression groups. Then, we also explored the correlation of B3GNT3 expression with gene markers of common tumor-infiltrating immune cells, including T cell (general), CD8 + T cell, Th1 cell, Tfh cell, Treg cell, Th17 cell, T cell exhaustion, B-cells, Natural killer cell, Neutrophils and Mast cells. The "correlation" module of an online database, the Gene Expression Profiling Interactive Analysis (GEPIA) (http://gepia.cancer-pku.cn/index.html), was employed to realize the analyses[15]. By this "correlation" module, the results can be presented as scatter plots of a defined gene pair from PAAD tumor alongside the Spearman's $\mathrm{R}$ and statistical significance. $P$-value $<$ 0.01 was determined as the threshold. All these cell type markers were well previously reported[15, 16].

\section{Gene set enrichment analysis}

Gene set enrichment analysis (GSEA) is a powerful analytical method for interpreting gene expression data by determining statistically significant differences between biological states in prior defined gene-sets[17]. In this study, GSEA software package v2.1.0 (Broad Institute, MIT, Cambridge, MA, USA) was downloaded and carried out to elucidate the potential mechanisms underlying the effect of B3GNT3 expression on PAAD patients. Gene set permutations were performed 1000 times for each analysis. A normalized enrichment score (NES) was calculated to sort the pathways enriched in each phenotype. Gene sets with a $P$-value $<0.05$ and false discovery rate (FDR) q-value $<0.05$ were regarded as statistically significant.

\section{Statistical analysis}

All statistical analyses were based on R program 4.0.3 version and attached packages. The correlations between expression of B3GNT3 and clinicopathologic parameters were analyzed using the Wilcoxon rank sum test, Chi-square test, Fisher's exact tests and Student's tests. The median value of B3GNT3 expression level was used to divide PAAD patients into high-expression and lowexpression groups. $P$ value $<0.05$ was considered statistically significant in this study.

\section{Results}

\section{Elevated expression of B3GNT3 in PAAD}


We first investigated the expression pattern of B3GNT3 in normal tissues using RNA-sequencing data from GTEx. We found that expression of B3GNT3 was low in pancreas both in male and female group (Figure A). Then, data extracted from TCGA database revealed that B3GNT3 expression was significantly higher in tumor tissues of PAAD patients $(N=178)$ compared to matched tumoradjacent normal tissues of TCGA $(\mathrm{N}=4)$ and GTEx normal tissues $(\mathrm{N}=167)(P<0.05$, Figure $\mathrm{B} 1, \mathrm{~B} 2)$. Finally, we assessed the expression of B3GNT3 in GSE62452 dataset. The results also showed an obvious increase in the expression of B3GNT3 in tumor tissues $(N=61)$ compared with tumor-adjacent normal tissues $(N=69)(P<0.05$, Figure B3, B4).

\section{Correlation of B3GNT3 expression level with clinicopathologic parameters of PAAD patients}

The clinicopathologic data of PAAD patients in TCGA were presented in Table 1. Then, we investigated the correlation between B3GNT3 expression and routine clinicopathologic characteristics, respectively. The results implied that patients with advanced histologic grade were observed more frequently in B3GNT3 high-expression group $(P<0.05)$. And its expression was higher in advanced stage (stage $\square$ Vs stage $\square, P<0.05)$. However, because of the small sample size $(N=7)$, no significant differences were found in cases of stage $\square$ and $\square$ patients. There were no significant correlations between B3GNT3 expression and other clinicopathologic parameters, such as age, gender, race, family history of cancer, alcohol consumption, maximum tumor dimension, tumor location, $\mathrm{N}$ status, or $\mathrm{M}$ status in tumor tissues of PAAD patients. The results were summarized in Figure $\mathrm{C} 1-\mathrm{C} 4$.

\section{Role of B3GNT3 differential expression in PAAD Prognosis}

Kaplan-Meier survival curves were performed to investigate the correlation between B3GNT3 expression and survival outcomes of PAAD cohorts with TCGA survival information available. The results revealed worse 1-year, 3-year and 5-year OS in PAAD patients with B3GNT3 higher expression levels compared to its lower expression levels (Figure D1-D3). Cases with higher B3GNT3 expression showed shorter median survival time compared with cases with lower B3GNT3 expression (15.87 vs 23.17 months; log-rank $P=$ 0.001). Then, we performed univariate Cox proportional hazards regression analysis to explore the associations of differentially expressed B3GNT3 and other clinicopathologic parameters with patient prognosis. The results displayed that B3GNT3, age, histologic grade, T status, $\mathrm{N}$ status, tumor location, Number of positive lymphnodes and residual tumor were associated with overall survival $(P$ value $<0.05)$ (Table 2). Using these prognostic factors, we conducted multivariate Cox proportional hazards regression analysis, of which only B3GNT3 and tumor location were found to be coupled to the overall survival of PAAD patients. The results were shown in Table 2 and Figure $\mathrm{E}$.

\section{Table 1}

The clinicopathologic data of PAAD patients in TCGA. 


\begin{tabular}{|c|c|c|c|}
\hline Clinical parameters & Variable & Total[176! & Percentage $(\%)$ \\
\hline \multirow[t]{2}{*}{ Age } & $\leq 60$ & 58 & 32.95 \\
\hline & $>60$ & 118 & 67.05 \\
\hline \multirow[t]{2}{*}{ Gender } & Male & 98 & $55.68 \%$ \\
\hline & Female & 78 & $44.32 \%$ \\
\hline \multirow[t]{4}{*}{ Race } & Asian & 11 & $6.25 \%$ \\
\hline & White & 155 & $88.07 \%$ \\
\hline & Black or African American & 6 & $3.41 \%$ \\
\hline & NA & 4 & $2.27 \%$ \\
\hline \multirow[t]{3}{*}{ Family history of cancer } & NO & 47 & $26.70 \%$ \\
\hline & Yes & 62 & $35.23 \%$ \\
\hline & NA & 67 & $38.07 \%$ \\
\hline \multirow[t]{3}{*}{ History of chronic pancreatitis } & NO & 127 & $72.16 \%$ \\
\hline & Yes & 13 & $7.39 \%$ \\
\hline & NA & 36 & $20.45 \%$ \\
\hline \multirow[t]{3}{*}{ History of diabetes } & NO & 107 & $60.80 \%$ \\
\hline & Yes & 38 & $21.59 \%$ \\
\hline & NA & 31 & $17.61 \%$ \\
\hline \multirow[t]{3}{*}{ Alcohol consumption } & NO & 64 & $36.36 \%$ \\
\hline & Yes & 100 & $56.82 \%$ \\
\hline & NA & 12 & $6.82 \%$ \\
\hline \multirow[t]{3}{*}{ Tumor location } & Head & 137 & $77.84 \%$ \\
\hline & Body/tail & 28 & $15.91 \%$ \\
\hline & Other & 11 & $6.25 \%$ \\
\hline \multirow[t]{3}{*}{ Maximum tumor dimension } & $\leq 4 \mathrm{~cm}$ & 109 & 61.93 \\
\hline & $>4 \mathrm{~cm}$ & 54 & 30.69 \\
\hline & NA & 13 & 7.38 \\
\hline \multirow[t]{5}{*}{ Histologic grade } & $\mathrm{G} 1$ & 30 & $17.05 \%$ \\
\hline & $\mathrm{G} 2$ & 94 & $53.41 \%$ \\
\hline & G3 & 48 & $27.27 \%$ \\
\hline & G4 & 2 & $1.14 \%$ \\
\hline & NA & 2 & $1.14 \%$ \\
\hline \multirow[t]{3}{*}{ T status } & $\mathrm{T} 1+\mathrm{T} 2$ & 31 & $17.61 \%$ \\
\hline & $\mathrm{T} 3+\mathrm{T} 4$ & 143 & $81.25 \%$ \\
\hline & NA & 2 & $1.14 \%$ \\
\hline \multirow[t]{2}{*}{$\mathrm{N}$ status } & NO & 49 & $27.84 \%$ \\
\hline & N1 & 122 & $69.32 \%$ \\
\hline
\end{tabular}

Page 5/13 


\begin{tabular}{|c|c|c|c|}
\hline & NA & 5 & $2.84 \%$ \\
\hline \multirow[t]{3}{*}{ M status } & MO & 79 & $44.89 \%$ \\
\hline & M1 & 4 & $2.27 \%$ \\
\hline & NA & 93 & $52.84 \%$ \\
\hline \multirow[t]{5}{*}{ Stage } & I & 21 & $11.93 \%$ \\
\hline & $\|$ & 145 & $82.39 \%$ \\
\hline & III & 3 & $1.70 \%$ \\
\hline & IV & 4 & $2.27 \%$ \\
\hline & NA & 1 & $0.57 \%$ \\
\hline
\end{tabular}

Table 2

Univariate and multivariate Cox regression analysis of overall survival in PAAD patients.

\begin{tabular}{|lllllllll|l|}
\hline & \multicolumn{3}{l}{ Univariate Cox regression } & \multicolumn{5}{c|}{ Multivariate Cox regression } \\
\hline Factors & HR & HR.95L & HR.95H & $P$ value & HR & HR.95L & HR.95H & $P$ value \\
\hline Gender & 0.914 & 0.595 & 1.406 & 0.683 & 0.657 & 0.399 & 1.083 & 0.099 \\
\hline Age & 1.031 & 1.008 & 1.054 & $0.008^{*}$ & 1.021 & 0.998 & 1.044 & 0.0698 \\
\hline G & 1.399 & 1.036 & 1.889 & $0.028^{*}$ & 1.157 & 0.834 & 1.606 & 0.382 \\
\hline T & 1.573 & 1.009 & 2.452 & $0.045^{*}$ & 1.011 & 0.522 & 1.960 & 0.973 \\
\hline N & 2.107 & 1.235 & 3.595 & $0.006^{*}$ & 1.187 & 0.609 & 2.313 & 0.615 \\
\hline Stage & 1.138 & 0.513 & 2.527 & 0.750 & 0.627 & 0.184 & 2.136 & 0.456 \\
\hline Tumor location & 0.652 & 0.458 & 0.929 & $0.018^{*}$ & 0.588 & 0.389 & 0.887 & $0.011 *$ \\
\hline Maximum tumor dimension & 0.986 & 0.878 & 1.108 & 0.818 & 0.998 & 0.833 & 1.196 & 0.984 \\
\hline Number of positive lymphnodes & 1.062 & 1.006 & 1.122 & $0.030^{*}$ & 1.101 & 1.001 & 1.211 & 0.048 \\
\hline Residual tumor & 1.799 & 1.156 & 2.801 & $0.009 *$ & 1.401 & 0.874 & 2.246 & 0.162 \\
\hline B3GNT3 & 1.386 & 1.121 & 1.713 & $0.002^{*}$ & 1.713 & 1.262 & 2.325 & 0.0005 * \\
\hline
\end{tabular}

\section{Correlation between B3GNT3 expression and tumor-infiltrating immune cells}

As is known, the microenvironment of PAAD is characterized by a dense stromal compartment and the tumor-infiltrating immune cells (TIICs) is a vital component of stromal. Prior published studies indicated that tumor-infiltrating lymphocyte levels might influence the prognosis of pancreatic cancer patients $[18,19]$. Hence, we also explored the relationship between B3GNT3 expression level and immune infiltration based on TCGA dataset using CIBERSORT algorithm. According to median value of the B3GNT3 expression level, 124 PAAD samples finally met screening criterion and were divided into high-expression and low-expression groups. The results showed that a total of five kinds of immune cells were associated with the B3GNT3 expression level. Among them, naive B cells, CD8 T cells, and CD4 memory activated T cells activated negatively correlated with B3GNT3 expression. In contrast, activated NK cells and Macrophages M0 cells positively correlated with B3GNT3 expression (Figure F).

Finally, to furtherly understand the possible role of B3GNT3 in tumor-infiltrating immune cells, we analyzed the association between B3GNT3 expression and multiple immune marker genes of tumor-infiltrating immune cells using the "correlation" module of GEPIA (Table 3). The results suggested that the expression of B3GNT3 negatively correlated with almost all marker sets of different immune 
cells in PAAD samples, including CD2, CD3D, CD3E and CD6 of T cell (general), CD8A and CD8B of CD8 + T cell, TBX21 of Th1 cell, FOXP3 of Treg cell, STAT3 and IL17A of Th17 cell, PD-1, CTLA4, LAG3 and IM-3 of T cell exhaustion, etc. The details of results were presented in Table 5 along with Spearman correlation coefficient and $p$ value. Hence, the above results indicated that there existed potential negative correlation between B3GNT3 expression and immune infiltration in PAAD.

\section{Table 3}

Association between B3GNT3 expression and gene markers of tumor-infiltrating immune cells using "correlation" module of GEPIA.

\begin{tabular}{|c|c|c|c|}
\hline & Gene markers & $\mathrm{R}$ & $P$ \\
\hline \multirow[t]{4}{*}{ T cell (general) } & $\mathrm{CD} 2$ & -0.38 & $1.3 e-07 *$ \\
\hline & CD3D & -0.29 & $8 e-05^{\star}$ \\
\hline & CD3E & -0.33 & $6.4 \mathrm{e}-06^{*}$ \\
\hline & CD6 & -0.29 & $6.5 e-05^{\star}$ \\
\hline \multirow[t]{2}{*}{ CD8+ T cell } & CD8A & -0.38 & $1.5 \mathrm{e}-07 *$ \\
\hline & CD8B & -0.36 & $8.7 e-07 *$ \\
\hline Th1 & TBX21 & -0.38 & $1.3 e-07 *$ \\
\hline Tfh & BCL6 & 0.16 & $0.033^{*}$ \\
\hline Treg cell & FOXP3 & -0.28 & $0.00016^{*}$ \\
\hline \multirow[t]{2}{*}{ Th17 } & STAT3 & -0.02 & 0.8 \\
\hline & IL17A & -0.12 & 0.12 \\
\hline \multirow[t]{4}{*}{ T cell exhaustion } & PD-1 & -0.3 & $4.4 \mathrm{e}-05^{\star}$ \\
\hline & CTLA4 & -0.31 & $2.1 \mathrm{e}-05^{\star}$ \\
\hline & LAG3 & -0.21 & $0.0057^{\star}$ \\
\hline & TIM-3 & -0.25 & $0.00074 *$ \\
\hline \multirow[t]{3}{*}{ B-cells } & BLK & -0.25 & $0.00078 *$ \\
\hline & CD19 & -0.23 & $0.0021^{\star}$ \\
\hline & FCRL2 & -0.22 & $0.0028^{\star}$ \\
\hline \multirow[t]{3}{*}{ Natural killer cell } & XCL1 & -0.064 & 0.39 \\
\hline & XCL2 & -0.3 & $3.7 e-05^{\star}$ \\
\hline & NCR1 & -0.16 & $0.033^{*}$ \\
\hline \multirow[t]{3}{*}{ Neutrophils } & FPR1 & -0.28 & $0.00012^{*}$ \\
\hline & SIGLEC5 & -0.24 & $0.0012^{\star}$ \\
\hline & CSF3R & -0.15 & 0.05 \\
\hline \multirow[t]{5}{*}{ Mast cells } & TPSB2 & -0.16 & $0.036^{*}$ \\
\hline & TPSAB1 & -0.1 & 0.17 \\
\hline & СРАЗ & -0.2 & $0.0082^{*}$ \\
\hline & MS4A2 & -0.24 & $0.0014^{\star}$ \\
\hline & $\mathrm{HDC}$ & -0.23 & $0.0018^{*}$ \\
\hline
\end{tabular}

Page $7 / 13$ 


\section{Functional enrichment analysis for B3GNT3}

To explore the potential molecular mechanisms of B3GNT3, GSEA was conducted to compare the B3GNT3 high-expression group with the low-expression group in PAAD patients of the whole set. GSEA demonstrated significant differences (FDR $<0.05, N O M P$ value $<0.05$ ) in enrichment of KEGG and GO collection. Due to the limitations of space, only twenty leading pathways of high and low expression were listed in Table 4. Nine KEGG items including adheres junction, ubiquitin mediated proteolysis, ERBB signaling pathway, basal transcription factors, pancreatic cancer, pathways in cancer, p53 signaling pathway, TGF beta signaling pathway and tight junction showed significantly differential enrichment in B3GNT3 high expression phenotype, one KEGG item of oxidative phosphorylation showed significantly differential enrichment in B3GNT3 low expression phenotype based on NES, NOM $P$-value, and FDR value (Figure G1). Ten GO items including nuclear receptor binding, cadherin binding, negative regulation of ubiquitin dependent protein catabolic process, response to epidermal growth factor, p53 binding, intracellular receptor signaling pathway, positive regulation of intracellular protein transport, regulation of protein export from nucleus, negative regulation of cellular protein catabolic process, and cell cycle G1 S phase transition were revealed significantly differential enrichment in B3GNT3 high expression phenotype and there was no signal pathway in low B3GNT3 expression based on NES, NOM P-value, and FDR value (Figure G2). All the results suggested the potential role of B3GNT3 in the development of PAAD.

\section{Table 4}

Gene sets enriched in the B3GNT3 high- and low-expression phenotype. 


\begin{tabular}{|c|c|c|c|c|}
\hline Gen set name & ES & NES & $\begin{array}{l}\text { NOM } \\
p \text {-val }\end{array}$ & $\begin{array}{l}\text { FDR } \\
q \text {-val }\end{array}$ \\
\hline \multicolumn{5}{|l|}{ High B3GNT3 expression phenotype } \\
\hline KEGG_ADHERENS_JUNCTION & 0.626 & 2.129 & 0.000 & 0.006 \\
\hline KEGG_UBIQUITIN_MEDIATED_PROTEOLYSIS & 0.418 & 1.969 & 0.002 & 0.020 \\
\hline KEGG_ERBB_SIGNALING_PATHWAY & 0.517 & 1.963 & 0.000 & 0.019 \\
\hline KEGG_BASAL_TRANSCRIPTION_FACTORS & 0.568 & 1.928 & 0.002 & 0.019 \\
\hline KEGG_PANCREATIC_CANCER & 0.589 & 1.918 & 0.002 & 0.017 \\
\hline KEGG_PATHWAYS_IN_CANCER & 0.494 & 1.886 & 0.002 & 0.020 \\
\hline KEGG_P53_SIGNALING_PATHWAY & 0.569 & 1.863 & 0.000 & 0.026 \\
\hline KEGG_TGF_BETA_SIGNALING_PATHWAY & 0.550 & 1.765 & 0.013 & 0.044 \\
\hline KEGG_TIGHT_JUNCTION & 0.486 & 1.940 & 0.000 & 0.018 \\
\hline GO_NUCLEAR_RECEPTOR_BINDING & 0.589 & 2.345 & 0.000 & 0.007 \\
\hline GO_CADHERIN_BINDING & 0.574 & 2.312 & 0.000 & 0.007 \\
\hline GO_NEGATIVE_REGULATION_OF_UBIQUITIN_DEPENDENT_PROTEIN_CATABOLIC_PROCESS & 0.521 & 2.283 & 0.000 & 0.010 \\
\hline GO_RESPONSE_TO_EPIDERMAL_GROWTH_FACTOR & 0.652 & 2.207 & 0.000 & 0.018 \\
\hline GO_P53_BINDING & 0.548 & 2.169 & 0.000 & 0.020 \\
\hline GO_INTRACELLULAR_RECEPTOR_SIGNALING_PATHWAY & 0.507 & 2.139 & 0.000 & 0.016 \\
\hline GO_POSITIVE_REGULATION_OF_INTRACELLULAR_PROTEIN_TRANSPORT & 0.461 & 2.128 & 0.000 & 0.013 \\
\hline GO_REGULATION_OF_PROTEIN_EXPORT_FROM_NUCLEUS & 0.612 & 2.181 & 0.000 & 0.020 \\
\hline GO_NEGATIVE_REGULATION_OF_CELLULAR_PROTEIN_CATABOLIC_PROCESS & 0.456 & 2.170 & 0.000 & 0.023 \\
\hline GO_CELL_CYCLE_G1_S_PHASE_TRANSITION & 0.516 & 2.105 & 0.000 & 0.014 \\
\hline \multicolumn{5}{|l|}{ Low B3GNT3 expression phenotype } \\
\hline KEGG_OXIDATIVE_PHOSPHORYLATION & -0.547 & -2.224 & 0.000 & 0.001 \\
\hline
\end{tabular}

ES: enrichment score; NES: normalized enrichment score; NOM: nominal; FDR: false discovery rate. Gene sets with a $p$-value $<0.05$ and false discovery rate (FDR) q-value $<0.05$ were regarded as statistically significant.

\section{Discussion}

In this study, we performed a retrospective analysis on 312 PAAD patients to illustrate the potential association of B3GNT3 and PAAD. As a result, we observed that B3GNT3 expression differed in normal and tumor tissues of PAAD. The expression of B3GNT3 was obviously up-regulated in tumor tissues compared with normal tissues both in TCGA and GEO datasets. Meanwhile, increased B3GNT3 expression significantly correlated with histologic grade and unfavorable prognosis. And the multivariate cox regression analyses suggested that B3GNT3 expression was an independent prognostic factor for PAAD patients. According to our knowledge, there was limited reports concerning the impact of B3GNT3 on PAAD.

0-glycosylation is one of the major post-translational modifications of proteins. O-glycans was not only presented on the surface of normal cells, but also the tumors. Prior reports have well studied that O-glycans participated in various biological and cellular signaling, including immune modulation, signal transduction, protein folding, tumorigenesis, etc.[20-23]. As an 0glycosyltransferases, B3GNT3 should transfer donor substrate sugars via a $\beta 1,3$-linkage and get involved in the development and 
progression of tumors. In 2018, a study indicated that elevated B3GNT3 expression played an important role in the maintenance of stemness in pancreatic cancer stem cells (PCSCs). Based on molecular and functional assays, the results revealed that B3GNT3 could modulate CSC markers including CD44v6, ESA, SOX2 and OCT3/4 and influence the self-renewal potential of PCSCs[24]. But, a later study from their group, regarding glycosyltransferases to PAAD pathogenesis, implicated that B3GNT3 negatively regulated the proliferation, migration and stem cell markers in vitro[25]. Therefore, B3GNT3 likely acted as a tumor-promoting role but the clear mechanisms were still unknown. Interestingly, as an 0-glycosyltransferases, the single nucleotide polymer-phisms (SNPs) of B3GNT3 also might affect levels of circulating tumor biomarkers including CA19-9, CEA and AFP. By SNP-defined ranges for each tumor marker, diagnostic specificity and sensitivity of CA19-9 were improved in the identification of patients with PAAD [26, 27]. As is known, CA19-9 shows many shortcomings of unsatisfactory diagnostic accuracy in the routine management of PAAD. And about 10-15\% of PAAD patients do not secrete CA19-9 due to their Lewis antigen status. Hence, B3GNT3 may be a potential biomarker and when in combination with CA19-9 might provide additional information for the screening and diagnosis of PAAD.

The tumor microenvironment (TME) consists of various cell types and extracellular component, which densely surrounds tumor cells. Tumor infiltrating immune cells (TIICs) are important components of TME and mainly include T cells, B cells, macrophages, dendritic cells (DCs), and natural killer (NK) cells, etc. TIICs play an important role in tumor initiation, progression and metastasis by regulating antitumor immune responses and remodeling an immunosuppressive microenvironment. Surrounded by densely immunosuppressive micro-environment, tumor cells finally escaped from immune surveillance and proliferated rapidly. Although the underlying mechanisms remain indefinite, evidence has shown that TILs proportion was a positive prognostic factor in various tumors[28, 29]. Based on RNA expression analyzed by CIBERSORT, one study indicated that higher levels of activated memory CD4 + T cells, activated mast cells and activated NK cells were associated with favorable overall survival in cervical cancer[30]. PAAD is characterized by the dense fibrotic tumor stroma and the immunosuppressive microenvironment, which inhibit the infiltration of immune cells and make common immune therapy ineffectiveness[31]. Hence, PAAD is thought to be immune-quiescent. Increasing evidence has suggested that tumor-infiltrating immune cells were positively correlated with long-term survival. As well as TNM classification system, quantification of TIICs may be a possible tool for additional immunological subtype classification in PAAD patients $[32,19]$. In our study, we also analyzed the correlation between B3GNT3 expression and TIICs profiles in PAAD using CIBERSORT method and the "correlation" module of GEPIA. Our results revealed that there existed a negatively correlation between B3GNT3 expression level and infiltrating immune cells level. In addition, the "correlation" module analysis furtherly implicated the negative correlation between B3GNT3 expression and the multiple immune marker genes. Our results implicated that high B3GNT3 might lead to poor prognosis by hampering immune cells infiltrating from circulation into tumors. However, the possible molecular mechanism is unclear and further study are needed to address this issue.

According to functional enrichment analysis, B3GNT3 was mainly involved in various molecular signaling pathways including pathways in cancer, p53 signaling pathway, TGF beta signaling pathway, etc. Catabolic and transport processes of proteins were also enriched. Besides, ubiquitination and adheres were related to the B3GNT3 signature. All these biological functions were reported to be vital in the tumorigenesis and progression of PAAD[33-35]. Hence, biological analysis revealed B3GNT3 promoting the development of PAAD through multiple pathways.

Although our study found successfully the close relationship between B3GNT3 expression and PAAD, some limitations of this study should be acknowledged. Firstly, both TCGA and GEO are powerful databases with a comprehensive landscape of genomic alterations. But some of the shortcomings should not be ignored: (1) The sample sizes of PAAD are relatively small in TCGA and GEO dataset. For example, there were only seven cases of stage $\square$ and $\square$ patients in TCGA, which might lead to statistical errors in analyzing the correlation between B3GNT3 expression and stage. (2) Corresponding clinical information of PAAD patients is inadequate in GEO, which makes it impossible to analyze effect of B3GNT3 on clinicopathological factors. Secondly, our results were based on high-throughput RNA-sequencing profiles and computational algorithm. Although the practicability and accuracy of this method has been testified by many studies, further experiments in vivo and vitro are necessary to verify the results in the future.

\section{Conclusions}

In conclusion, B3GNT3 was identified to be overexpressed in PAAD tissues based on GTEx, TCGA and GEO databases. Increased B3GNT3 expression was closely correlated with worse OS in PAAD patients. Meanwhile, B3GNT3 expression level was negatively correlated with proportions of tumor-infiltrating immune cells. Hence, B3GNT3 might affect the infiltration of immune cells, and could act as a potential prognostic biomarker of PAAD.

Page 10/13 


\section{Declarations}

\section{Acknowledgements}

None.

Funding

None.

\section{Conflict of interest}

The authors declare that they have no conflict of interest.

\section{Ethics approval and availability of data and material}

Due to the public availability of data on GTEx, TCGA and GEO database, additional restriction or limitation was not required for this study.

\section{Author contributions[}

All authors contributed to the study conception and design. Long-Jiang Chen, Lu-Lu Zhai and Wei Wang performed data collection and analysis. The first draft of the manuscript was written by Long-Jiang Chen. Zhi-Gang Tang designed the study, performed critical revision and supervised all phases of the study. All authors were involved in the critical revision of the finial manuscript. All authors read and approved the final manuscript.

\section{References}

1. Siegel RL, Miller KD, Jemal A (2020) Cancer statistics, 2020. CA Cancer J Clin 70(1):7-30. https://doi.org/10.3322/caac.21590

2. Collaborators GBDPC (2019) The global, regional, and national burden of pancreatic cancer and its attributable risk factors in 195 countries and territories, 1990-2017: a systematic analysis for the Global Burden of Disease Study 2017. Lancet Gastroenterol Hepatol 4(12):934-947. https://doi.org/10.1016/S2468-1253(19)30347-4

3. Walter FM, Mills K, Mendonça SC, Abel GA, Basu B, Carroll N, Ballard S, Lancaster J, Hamilton W, Rubin GP, Emery JD (2016) Symptoms and patient factors associated with diagnostic intervals for pancreatic cancer (SYMPTOM pancreatic study): a prospective cohort study. Lancet Gastroenterol Hepatol 1(4):298-306. https://doi.org/10.1016/s2468-1253(16)30079-6

4. Sung H, Siegel RL, Rosenberg PS, Jemal A (2019) Emerging cancer trends among young adults in the USA: analysis of a population-based cancer registry. Lancet Public Health 4(3):e137-e147. https://doi.org/10.1016/s2468-2667(18)30267-6

5. Naudin S, Viallon V, Hashim D, Freisling H, Jenab M, Weiderpass E, Perrier F, McKenzie F, Bueno-de-Mesquita HB, Olsen A, Tjønneland A, Dahm CC, Overvad K, Mancini FR, Rebours V, Boutron-Ruault MC, Katzke V, Kaaks R, Bergmann M, Boeing H, Peppa E, Karakatsani A, Trichopoulou A, Pala V, Masala G et al (2020) Healthy lifestyle and the risk of pancreatic cancer in the EPIC study. Eur J Epidemiol 35(10):975-986. https://doi.org/10.1007/s10654-019-00559-6

6. Kirkegård J, Lund JL, Mortensen FV, Cronin-Fenton D (2020) Statins and pancreatic cancer risk in patients with chronic pancreatitis: A Danish nationwide population-based cohort study. Int J Cancer 146(3):610-616.

https://doi.org/10.1002/ijc.32264

7. Huang BZ, Pandol SJ, Jeon CY, Chari ST, Sugar CA, Chao CR, Zhang ZF, Wu BU, Setiawan VW (2020) New-Onset Diabetes, Longitudinal Trends in Metabolic Markers, and Risk of Pancreatic Cancer in a Heterogeneous Population. Clin Gastroenterol Hepatol 18(8):1812-1821.e1817. https://doi.org/10.1016/j.cgh.2019.11.043

8. Hu C, Hart SN, Polley EC, Gnanaolivu R, Shimelis H, Lee KY, Lilyquist J, Na J, Moore R, Antwi So, Bamlet WR, Chaffee KG, DiCarlo J, Wu Z, Samara R, Kasi PM, McWilliams RR, Petersen GM, Couch FJ (2018) Association Between Inherited Germline Mutations in Cancer Predisposition Genes and Risk of Pancreatic Cancer. Jama 319(23):2401-2409. https://doi.org/10.1001/jama.2018.6228

9. Sun Y, Liu T, Xian L, Liu W, Liu J, Zhou H (2020) B3GNT3, a Direct Target of miR-149-5p, Promotes Lung Cancer Development and Indicates Poor Prognosis of Lung Cancer. Cancer Manag Res 12:2381-2391. https://doi.org/10.2147/CMAR.S236565 
10. Zhang W, Hou T, Niu C, Song L, Zhang Y (2015) B3GNT3 Expression Is a Novel Marker Correlated with Pelvic Lymph Node Metastasis and Poor Clinical Outcome in Early-Stage Cervical Cancer. PLoS One 10(12):e0144360.

https://doi.org/10.1371/journal.pone.0144360

11. Ho WL, Che MI, Chou CH, Chang HH, Jeng YM, Hsu WM, Lin KH, Huang MC (2013) B3GNT3 expression suppresses cell migration and invasion and predicts favorable outcomes in neuroblastoma. Cancer Sci 104(12):1600-1608.

https://doi.org/10.1111/cas.12294

12. Cline MS, Craft B, Swatloski T, Goldman M, Ma S, Haussler D, Zhu J (2013) Exploring TCGA Pan-Cancer data at the UCSC Cancer Genomics Browser. Sci Rep 3:2652. https://doi.org/10.1038/srep02652

13. Ritchie ME, Phipson B, Wu D, Hu Y, Law CW, Shi W, Smyth GK (2015) limma powers differential expression analyses for RNAsequencing and microarray studies. Nucleic Acids Res 43(7):e47. https://doi.org/10.1093/nar/gkv007

14. Newman AM, Liu CL, Green MR, Gentles AJ, Feng W, Xu Y, Hoang CD, Diehn M, Alizadeh AA (2015) Robust enumeration of cell subsets from tissue expression profiles. Nat Methods 12(5):453-457. https://doi.org/10.1038/nmeth.3337

15. Wang W, Ren S, Wang Z, Zhang C, Huang J (2020) Increased expression of TTC21A in lung adenocarcinoma infers favorable prognosis and high immune infiltrating level. Int Immunopharmacol 78:106077. https://doi.org/10.1016/j.intimp.2019.106077

16. Danaher P, Warren S, Dennis L, D'Amico L, White A, Disis ML, Geller MA, Odunsi K, Beechem J, Fling SP (2017) Gene expression markers of Tumor Infiltrating Leukocytes. J Immunother Cancer 5:18. https://doi.org/10.1186/s40425-017-0215-8

17. Subramanian A, Tamayo P, Mootha VK, Mukherjee S, Ebert BL, Gillette MA, Paulovich A, Pomeroy SL, Golub TR, Lander ES, Mesirov JP (2005) Gene set enrichment analysis: a knowledge-based approach for interpreting genome-wide expression profiles. Proc Natl Acad Sci U S A 102(43):15545-15550. https://doi.org/10.1073/pnas.0506580102

18. D'Angelo A, Sobhani N, Roviello G, Bagby S, Bonazza D, Bottin C, Giudici F, Zanconati F, De Manzini N, Guglielmi A, Generali D (2019) Tumour infiltrating lymphocytes and immune-related genes as predictors of outcome in pancreatic adenocarcinoma. PLoS One 14(8):e0219566. https://doi.org/10.1371/journal.pone.0219566

19. Miksch RC, Schoenberg MB, Weniger M, Bösch F, Ormanns S, Mayer B, Werner J, Bazhin AV, D'Haese JG (2019) Prognostic Impact of Tumor-Infiltrating Lymphocytes and Neutrophils on Survival of Patients with Upfront Resection of Pancreatic Cancer. Cancers (Basel) 11 (1). https://doi.org/10.3390/cancers11010039

20. Itano N (2019) Implications of altered O-glycosylation in tumour immune evasion. J Biochem 165(5):387-390. https://doi.org/10.1093/jb/mvz003

21. Jiang Y, Liu Z, Xu F, Dong X, Cheng Y, Hu Y, Gao T, Liu J, Yang L, Jia X, Qian H, Wen T, An G (2018) Aberrant O-glycosylation contributes to tumorigenesis in human colorectal cancer. J Cell Mol Med 22(10):4875-4885. https://doi.org/10.1111/jcmm.13752

22. Oyama M, Kariya Y, Kariya Y, Matsumoto K, Kanno M, Yamaguchi Y, Hashimoto Y (2018) Biological role of site-specific Oglycosylation in cell adhesion activity and phosphorylation of osteopontin. Biochem J 475(9):1583-1595. https://doi.org/10.1042/bcj20170205

23. Varshney S, Stanley P (2018) Multiple roles for O-glycans in Notch signalling. FEBS Lett 592(23):3819-3834. https://doi.org/10.1002/1873-3468.13251

24. Barkeer S, Chugh S, Karmakar S, Kaushik G, Rauth S, Rachagani S, Batra SK, Ponnusamy MP (2018) Novel role of Oglycosyltransferases GALNT3 and B3GNT3 in the self-renewal of pancreatic cancer stem cells. BMC Cancer 18(1):1157. https://doi.org/10.1186/s12885-018-5074-2

25. Gupta R, Leon F, Thompson CM, Nimmakayala R, Karmakar S, Nallasamy P, Chugh S, Prajapati DR, Rachagani S, Kumar S, Ponnusamy MP (2020) Global analysis of human glycosyltransferases reveals novel targets for pancreatic cancer pathogenesis. Br J Cancer 122(11):1661-1672. https://doi.org/10.1038/s41416-020-0772-3

26. Abe T, Koi C, Kohi S, Song KB, Tamura K, Macgregor-Das A, Kitaoka N, Chuidian M, Ford M, Dbouk M, Borges M, He J, Burkhart R, Wolfgang CL, Klein AP, Eshleman JR, Hruban RH, Canto MI, Goggins M (2020) Gene Variants That Affect Levels of Circulating Tumor Markers Increase Identification of Patients With Pancreatic Cancer. Clin Gastroenterol Hepatol 18(5):1161-1169.e1165. https://doi.org/10.1016/j.cgh.2019.10.036

27. He M, Wu C, Xu J, Guo H, Yang H, Zhang X, Sun J, Yu D, Zhou L, Peng T, He Y, Gao Y, Yuan J, Deng Q, Dai X, Tan A, Feng Y, Zhang H, Min X, Yang X, Zhu J, Zhai K, Chang J, Qin X, Tan W et al (2014) A genome wide association study of genetic loci that 
influence tumour biomarkers cancer antigen $19-9$, carcinoembryonic antigen and a fetoprotein and their associations with cancer risk. Gut 63(1):143-151. https://doi.org/10.1136/gutjnl-2012-303434

28. Yang S, Liu T, Cheng Y, Bai Y, Liang G (2019) Immune cell infiltration as a biomarker for the diagnosis and prognosis of digestive system cancer. Cancer Sci 110(12):3639-3649. https://doi.org/10.1111/cas.14216

29. Chen B, Lai J, Dai D, Chen R, Li X, Liao N (2019) JAK1 as a prognostic marker and its correlation with immune infiltrates in breast cancer. Aging 11(23):11124-11135. https://doi.org/10.18632/aging.102514

30. Wang J, Li Z, Gao A, Wen Q, Sun Y (2019) The prognostic landscape of tumor-infiltrating immune cells in cervical cancer. Biomed Pharmacother 120:109444. https://doi.org/10.1016/j.biopha.2019.109444

31. Garg B, Giri B, Modi S, Sethi V, Castro I, Umland O, Ban Y, Lavania S, Dawra R, Banerjee S, Vickers S, Merchant NB, Chen SX, Gilboa E, Ramakrishnan S, Saluja A, Dudeja V (2018) NFkB in Pancreatic Stellate Cells Reduces Infiltration of Tumors by Cytotoxic T Cells and Killing of Cancer Cells, via Up-regulation of CXCL12. Gastroenterology 155(3):880-891.e888. https://doi.org/10.1053/j.gastro.2018.05.051

32. Nejati R, Goldstein JB, Halperin DM, Wang H, Hejazi N, Rashid A, Katz MH, Lee JE, Fleming JB, Rodriguez-Canales J, Blando J, Wistuba II, Maitra A, Wolff RA, Varadhachary GR, Wang H (2017) Prognostic Significance of Tumor-Infiltrating Lymphocytes in Patients With Pancreatic Ductal Adenocarcinoma Treated With Neoadjuvant Chemotherapy. Pancreas 46(9):1180-1187. https://doi.org/10.1097/mpa.0000000000000914

33. Li P, Cao G, Zhang Y, Shi J, Cai K, Zhen L, He X, Zhou Y, Li Y, Zhu Y, Xiong M, Wu Y (2020) FHL3 promotes pancreatic cancer invasion and metastasis through preventing the ubiquitination degradation of EMT associated transcription factors. Aging 12(1):53-69. https://doi.org/10.18632/aging.102564

34. Wang Z, Liu T, Xue W, Fang Y, Chen X, Xu L, Zhang L, Guan K, Pan J, Zheng L, Qin G, Wang T (2020) ARNTL2 promotes pancreatic ductal adenocarcinoma progression through TGF/BETA pathway and is regulated by miR-26a-5p. Cell Death Dis 11(8):692. https://doi.org/10.1038/s41419-020-02839-6

35. Tiwari A, Tashiro K, Dixit A, Soni A, Vogel K, Hall B, Shafqat I, Slaughter J, Param N, Le A, Saunders E, Paithane U, Garcia G, Campos AR, Zettervall J, Carlson M, Starr TK, Marahrens Y, Deshpande AJ, Commisso C, Provenzano PP, Bagchi A (2020) Loss of HIF1A From Pancreatic Cancer Cells Increases Expression of PPP1R1B and Degradation of p53 to Promote Invasion and Metastasis. Gastroenterology 159(5):1882-1897.e1885. https://doi.org/10.1053/j.gastro.2020.07.046 